\title{
Influence of surface roughness on subdominant pairing in $d$-wave superconductors
}

\author{
Thomas Lück*1 \\ ${ }^{1}$ Universität Augsburg, Institut für Physik, Theoretische Physik II, 86135 Augsburg, Germany
}

\begin{abstract}
Key words $d$-wave superconductivity, quasiclassical theory, time-reversal symmetry breaking. PACS 74.76.Bz, 74.50.+r

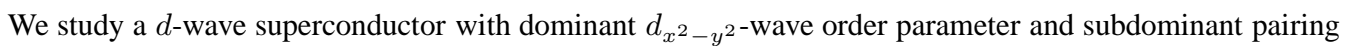
in either the $s$ - or the $d_{x y}$-wave channel near a surface. In particular we analyze the influence of surface roughness on the mixed order parameter which may break the time-reversal symmetry. We find that the subdominant component is suppressed by the roughness independent of its pairing symmetry; for very rough surfaces the subdominant component may even vanish completely. Additionally we discuss a possible realvalued admixture which counteracts the suppression of the $d_{x^{2}-y^{2}}$-wave order parameter at the surface.
\end{abstract}

There is an on-going discussion about the surface state of high- $T_{c}$ materials in the superconducting phase, which is commonly believed to exhibit $d$-wave symmetry in the bulk. In particular the question is whether a subdominant order parameter occurs at the surface which leads to a breaking of the time-reversal symmetry. Theoretical studies show that this is possible for low enough temperatures if the $d$-wave order parameter is strongly suppressed at the surface, and a subdominant pairing interaction exists [1] 2, 3]: Such a situation is realized, for example, if the surface is oriented in the [110] direction of a $d_{x^{2}-y^{2}}$ wave superconductor with subdominant pairing channels of the $s$-wave or the $d_{x y}$-wave type. This would manifest itself in the differential conductance of tunnel junctions as a splitting of the zero-bias peak which occurs for a tilted single-component $d_{x^{2}-y^{2}}$-wave order parameter [3]. Another experimental possibility is to study the magnetic field dependence of the differential conductance, which would also show anomalies due to a subdominant pairing interaction [4]. In this work we concentrate on the zero-field case.

In some experiments on high- $T_{c}$ superconductors a splitting of the zero-bias conductance peak was observed [5 6], which seems to confirm the existence of a subdominant order parameter. On the other hand, in numerous similar experiments no such splitting was visible [7, 8] 9]. It was shown experimentally for YBCO [6] that one reason for this discrepancy might be a different oxygen doping, i.e. different electronic properties of the material. We show here that these results can also be explained by surface roughness, as a rough surface can suppress a subdominant admixture of either $d_{x y}$-wave or $s$-wave symmetry. Moreover we demonstrate that in some cases a subdominant admixture to the order parameter occurs without breaking the time-reversal symmetry; then the spatial symmetry is modified near the surface.

To study the superconducting phase of high- $T_{c}$ superconductors we start with a few symmetry considerations. In conventional superconductors the gauge symmetry is broken only, and usually the order parameter is assumed to be isotropic $\left(\Delta\left(\mathbf{p}_{F}\right)=\Delta, \mathbf{p}_{F}\right.$ : Fermi momentum). In unconventional superconductors the lattice symmetry is broken in addition to the gauge symmetry, and the order parameter is anisotropic. In order to describe high- $T_{c}$ materials we consider a tetragonal lattice symmetry; note that we neglect possible orthorhombic distortions, present for example in YBCO. Then the order parameter can be

\footnotetext{
* E-mail: lueckt@physik.uni-augsburg.de, Phone: +49 821 5983255, Fax: +49 8215983262
} 
expanded as

$$
\Delta\left(\mathbf{p}_{F}\right)=-\sum_{i} \Delta_{i} \eta_{i}\left(\mathbf{p}_{F}\right)
$$

where $\eta_{i}$ are basis functions of the irreducible representations of the tetragonal space group, $D_{4 h}$. The lowest-order basis functions are given in Table 1 Since we neglect the weak dispersion in $c$-direction, we concentrate on three order parameter symmetries, namely $d_{x^{2}-y^{2}}, d_{x y^{-}}$and $s$-wave (see Fig. (1). In the following we will discuss a dominant $d_{x^{2}-y^{2}}$-wave order parameter, and a weaker pairing interaction in either the $s$-wave or the $d_{x y}$-wave channel.

Therefore we consider a superconductor with two (or more) possible order parameter components. Due to the pairing interaction each component has its individual critical temperature, $T_{c, i}$; i.e. in a bulk superconductor the $i^{\text {th }}$ order parameter component would become finite at $T_{i}$ if all other components were zero. The two cases of interest for us, namely a $d_{x^{2}-y^{2}}$-wave order parameter with $s$ - or $d_{x y}$-wave admixture, are discussed in Refs. [10] and [11], respectively, with the following results: If both components, the dominant and the subdominant, have a finite critical temperature $\left(T_{c, i}>0\right)$, it is possible that the dominant order parameter component with $T_{c, 1}$ suppresses the subdominant component, which then becomes finite at a lower temperature $\tilde{T}_{c, 2}<T_{c, 2}<T_{c, 1}$; alternatively it may happen that the subdominant pairing channel is completely suppressed, i.e. $\tilde{T}_{c, 2}=0$. The latter situation can be assumed for high- $T_{c}$ superconductors, where in the bulk only a $d_{x^{2}-y^{2}}$-wave order parameter is observed, while other order parameter components seem to be absent.

Although the subdominant pairing can be neglected in the bulk, it might become important near inhomogeneities, e.g. impurities or boundaries, which tend to suppress the dominant $d_{x^{2}-y^{2}}$-wave order parameter. At a specular surface, for example, a $d_{x^{2}-y^{2}}$-wave order parameter which is tilted by $45^{\circ}$ with respect to the surface normal is suppressed completely. In the following we will discuss the interplay between the suppression of the $d_{x^{2}-y^{2}}$-wave order parameter near an interface and the existence of a subdominant order parameter component in this region. In particular we study the influence of interface roughness, which also modifies the behavior of the $d_{x^{2}-y^{2}}$-wave component.

\begin{tabular}{lll}
$D_{4 h}:$ & basis functions & \\
\hline$A_{1 g}$ & $\eta_{1}(\mathbf{p})=1, p_{x}^{2}+p_{y}^{2}, p_{z}^{2}$ & (anisotropic) $s$-wave \\
$A_{2 g}$ & $\eta_{2}(\mathbf{p})=p_{x} p_{y}\left(p_{x}^{2}-p_{y}^{2}\right)$ & \\
$B_{1 g}$ & $\eta_{3}(\mathbf{p})=p_{x}^{2}-p_{y}^{2}$ & $d_{x^{2}-y^{2} \text {-wave }}$ \\
$B_{2 g}$ & $\eta_{4}(\mathbf{p})=p_{x} p_{y}$ & $d_{x y}$-wave \\
$E_{g}$ & $\eta_{5}(\mathbf{p})=\left(p_{x} p_{z}, p_{y} p_{z}\right)$ &
\end{tabular}

Table 1 Basis functions of lowest order for the group $D_{4 h}$.
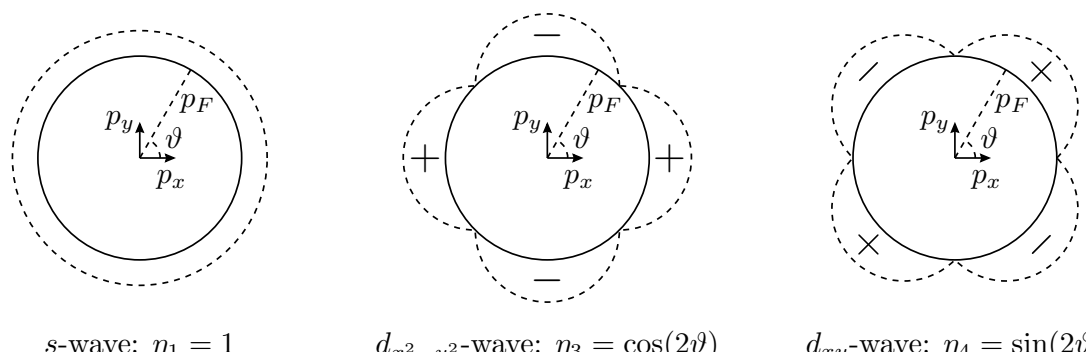

Fig. 1 Angular dependence of the order parameter for three different symmetries: $s-, d_{x^{2}-y^{2-}}$, and $d_{x y^{-}}$ wave. 
Here we apply the theory of quasiclassical Green's functions in thermal equilibrium [12, 13]. The Green's functions are determined by the Eilenberger equation,

$$
\left[\hat{\tau}_{3} E+e \hat{\tau}_{3} \mathbf{v}_{F} \cdot \mathbf{A}(\mathbf{r})+i \hat{\Delta}\left(\mathbf{p}_{F}, \mathbf{r}\right), \hat{g}\left(E, \mathbf{p}_{F} ; \mathbf{r}\right)\right]+i \mathbf{v}_{F} \cdot \partial_{\mathbf{r}} \hat{g}\left(E, \mathbf{p}_{F} ; \mathbf{r}\right)=0,
$$

supplemented by the normalization condition

$$
\left[\hat{g}\left(E, \mathbf{p}_{F} ; \mathbf{r}\right)\right]^{2}=\hat{1}
$$

Here $\hat{\tau}_{i}$ are the Pauli-matrices in Nambu-space. The (spin-singlet) order parameter

$$
\hat{\Delta}\left(\mathbf{p}_{F}, \mathbf{r}\right)=\left(\begin{array}{cc}
0 & \Delta\left(\mathbf{p}_{F}, \mathbf{r}\right) \\
\Delta^{*}\left(\mathbf{p}_{F}, \mathbf{r}\right) & 0
\end{array}\right)
$$

must be determined self-consistently via

$$
\hat{\Delta}\left(\mathbf{p}_{F}, \mathbf{r}\right)=-\pi \mathcal{N}_{0} T \sum_{\left|E_{n}\right|<E_{c}}\left\langle V\left(\mathbf{p}_{F}, \mathbf{p}_{F}^{\prime}\right) \hat{g}\left(i E_{n}, \mathbf{p}_{F}^{\prime} ; \mathbf{r}\right)\right\rangle_{\mathbf{p}_{F}^{\prime}},
$$

where $\langle\ldots\rangle_{\mathbf{p}_{F}^{\prime}}$ denotes an average over the Fermi surface (assumed to be spherical). The cut-off energy is $E_{c}$, and the normal-state density of states at the Fermi energy is denoted by $\mathcal{N}_{0}$ per spin. According to the above remarks on the order parameter symmetry we can expand the pairing interaction as follows:

$$
V\left(\mathbf{p}_{F}, \mathbf{p}_{F}^{\prime}\right)=-\sum_{i} V_{i} \eta_{i}\left(\mathbf{p}_{F}\right) \eta_{i}\left(\mathbf{p}_{F}^{\prime}\right)
$$

The current density can be determined from the Greeen's function via

$$
\mathbf{j}(\mathbf{r})=-i e \pi \mathcal{N}_{0} T \sum_{E_{n}} \operatorname{Tr}\left[\hat{\tau}_{3}\left\langle\mathbf{v}_{F} \hat{g}\left(i E_{n}, \mathbf{p}_{F} ; \mathbf{r}\right)\right\rangle_{\mathbf{p}_{F}}\right]
$$

and the angle-resolved density of states is given by

$$
\mathcal{N}\left(E, \mathbf{p}_{F} ; \mathbf{r}\right)=\frac{\mathcal{N}_{0}}{2} \operatorname{Re}\left\{\operatorname{Tr}\left[\hat{\tau}_{3} \hat{g}\left(E+i 0_{+}, \mathbf{p}_{F} ; \mathbf{r}\right)\right]\right\} .
$$

The density of states at the surface is connected to the differential tunnel conductance by the relation [14]

$$
G(V)=A e^{2}\left\langle v_{F, x} \mathcal{T}\left(\mathbf{p}_{F}\right) \mathcal{N}\left(e V, \mathbf{p}_{F} ; 0\right)\right\rangle_{\mathbf{p}_{F}},
$$

where $\mathcal{T}\left(\mathbf{p}_{F}\right)=\mathcal{T}_{0} p_{F, x}^{2} / p_{F}^{2}\left(\mathcal{T}_{0} \ll 1\right)$ is the angle-dependent transparency, and $A$ is the cross-section of the tunneling contact; this results in the normal state resistance $R_{N}^{-1}=4 A e^{2} \mathcal{N}_{0} v_{F} \mathcal{T}_{0} / 3 \pi$.

The quasiclassical approach is valid only on scales which are large compared to the Fermi wavelength, and is not directly applicable near interfaces. This problem must be circumvented using boundary conditions for the Green's functions. For specular interfaces, i.e. when the momentum parallel to the interface is conserved, one can use Zaitsev's boundary conditions [15]; these can be extended to include interface roughness [16 17 18, 19]. We use here an approach which was introduced by Shelankov and Ozana [20], in which the properties of the interface are described by a scattering matrix.

In order to describe interface roughness, which is present on the atomic scale, we choose the scattering matrix to be a random unitary matrix [16, 14, 21]. Here the only relevant roughness parameter is the probability for specular scattering, $|u|^{2}$. A rough interface leads to a reduced weight for specular scattering, $|u|^{2}<1$, and with a probability of $1-|u|^{2}$ a particle is scattered randomly into any other direction. This model is described in detail in [14, 21]. The disorder averaged quantities are denoted by $\langle\ldots\rangle$. 

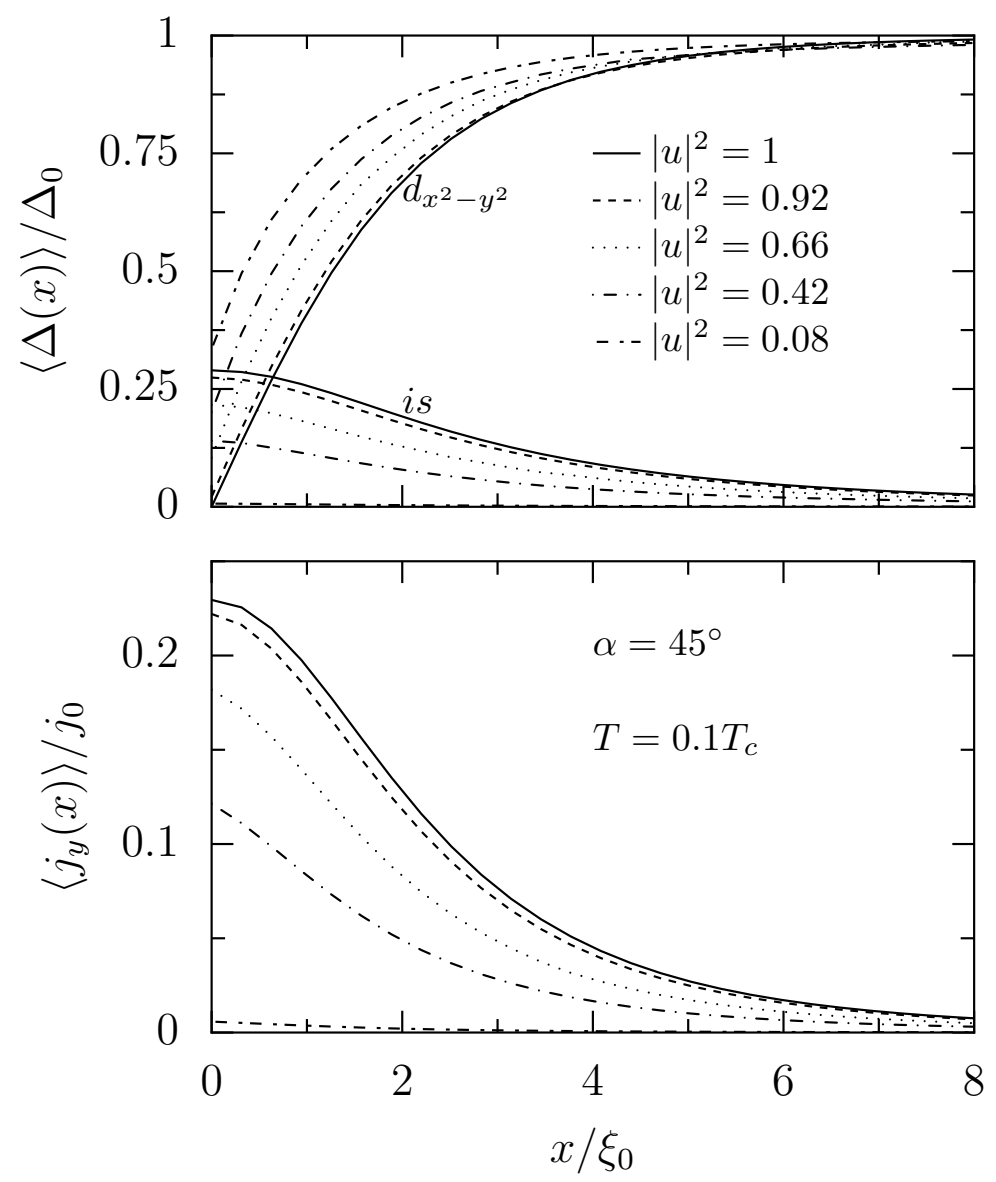

Fig. 2 Dominant $d_{x^{2}-y^{2}}$-wave order parameter with (imaginary) subdominant $s$-wave component, for $\alpha=45^{\circ}$ at $T=0.1 T_{c}$ (upper panel), and the resulting current flowing parallel to the surface (lower panel); $\Delta_{0}=2.14 T_{c}, \xi_{0}=v_{F} / \pi \Delta_{0}$, and $j_{0}=2 e \mathcal{N}_{0} v_{F} \Delta_{0}$.

The superconductor is described by a dominant $d_{x^{2}-y^{2}}$-wave order parameter with a critical temperature $T_{c}$, and a second order parameter component of either $s$ - or $d_{x y}$-wave type with an individual critical temperature $T_{c, 2}=0.3 T_{c}$; this choice ensures that in the bulk only a $d_{x^{2}-y^{2}}$-wave order parameter is present. The surface normal is in the $x$-direction, and we assume translational invariance in the $y$-direction (parallel to the surface).

The behavior of a single-component $d_{x^{2}-y^{2}}$-wave superconductor near a surface is well known [16, 17 18. 19 14]. The following results are of particular importance for our purpose: If the order parameter is tilted with respect to the surface normal, trajectories exist where the order parameter changes its sign due to surface scattering; this leads to a zero-bias peak in the differential conductance of tunnel contacts, and a suppression of the order parameter near the surface. Without surface roughness and for a tilting angle of $45^{\circ}$ the order parameter at the surface is zero. Surface disorder leads to a finite value of the order parameter, and in most models to a broadened zero-bias conductance peak. Furthermore, even an untilted $d_{x^{2}-y^{2}}$-wave order parameter is suppressed near a rough surface [14].

First we focus on a $d_{x^{2}-y^{2}}$-wave superconductor with a subdominant pairing in the $s$-wave channel; the order parameter is rotated by $45^{\circ}$ with respect to the surface normal. Here it is important to note that an isotropic $s$-wave order parameter itself is inert against scattering at a rough surface. Without roughness 

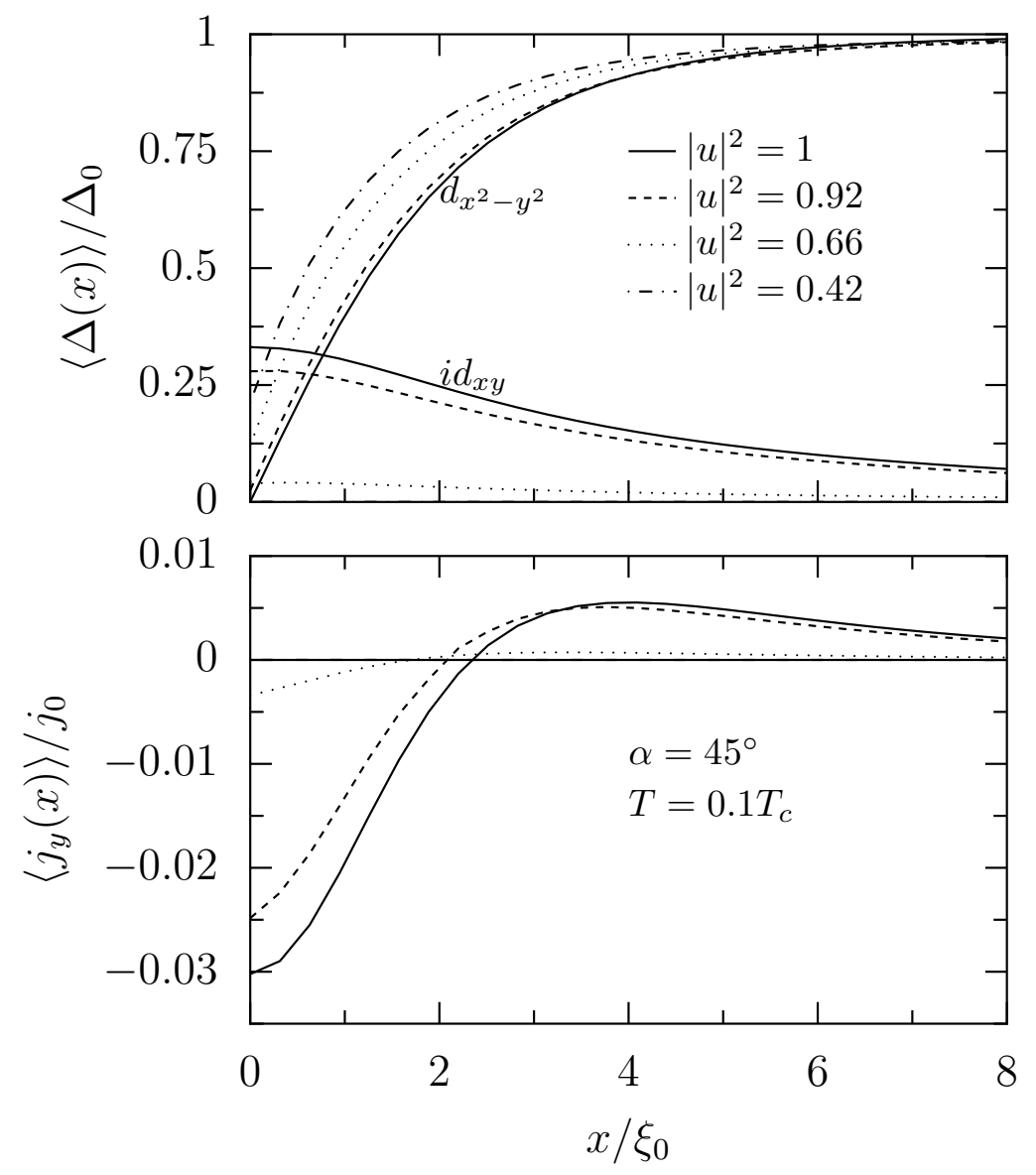

Fig. 3 Dominant $d_{x^{2}-y^{2}}$-wave order parameter with (imaginary) subdominant $d_{x y}$-wave component, for $\alpha=45^{\circ}$ at $T=0.1 T_{c}$ (upper panel), and the resulting current flowing parallel to the surface (lower panel); $\Delta_{0}=2.14 T_{c}, \xi_{0}=v_{F} / \pi \Delta_{0}$, and $j_{0}=2 e \mathcal{N}_{0} v_{F} \Delta_{0}$.

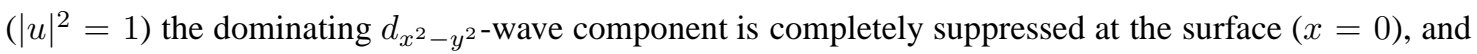
a finite $s$-wave admixture occurs near the boundary with a relative phase $\varphi= \pm \pi / 2$ (Fig. 2). This state has a spontaneously broken time-reversal symmetry near the surface. As a consequence a current flowing in $y$-direction is present (Fig. 22), and a splitting of the zero-bias conductance peak is found (Fig. (4). These are well-known results for the clean case [1, 2, 3, 22].

For a rough surface the $d_{x^{2}-y^{2}}$-wave component recovers and has a finite value at the surface; this in turn leads to a suppression of the subdominant component, the suppression becoming stronger with increasing roughness (Fig. 2). As a consequence the splitting of the zero-bias conductance peak and the parallel current are reduced. For very strong roughness $\left(|u|^{2} \lesssim 0.08\right)$ no subdominant order parameter is observed. Therefore the differential conductivity is no longer affected by the subdominant pairing, and no parallel current exists.

Next we consider the situation with a subdominant $d_{x y}$-wave component. In the clean case we find similar results as above, since the $45^{\circ}$-rotated $d_{x y}$-wave order parameter is not suppressed by the surface. Therefore a finite $d_{x y}$-wave admixture with a relative phase of $\varphi= \pm \pi / 2$ exists near the surface, and the resulting state exhibits broken time-reversal symmetry. Consequently a current in $y$-direction flows near the surface (Fig. 3), and the zero-bias conductance peak splits (Fig.4). 

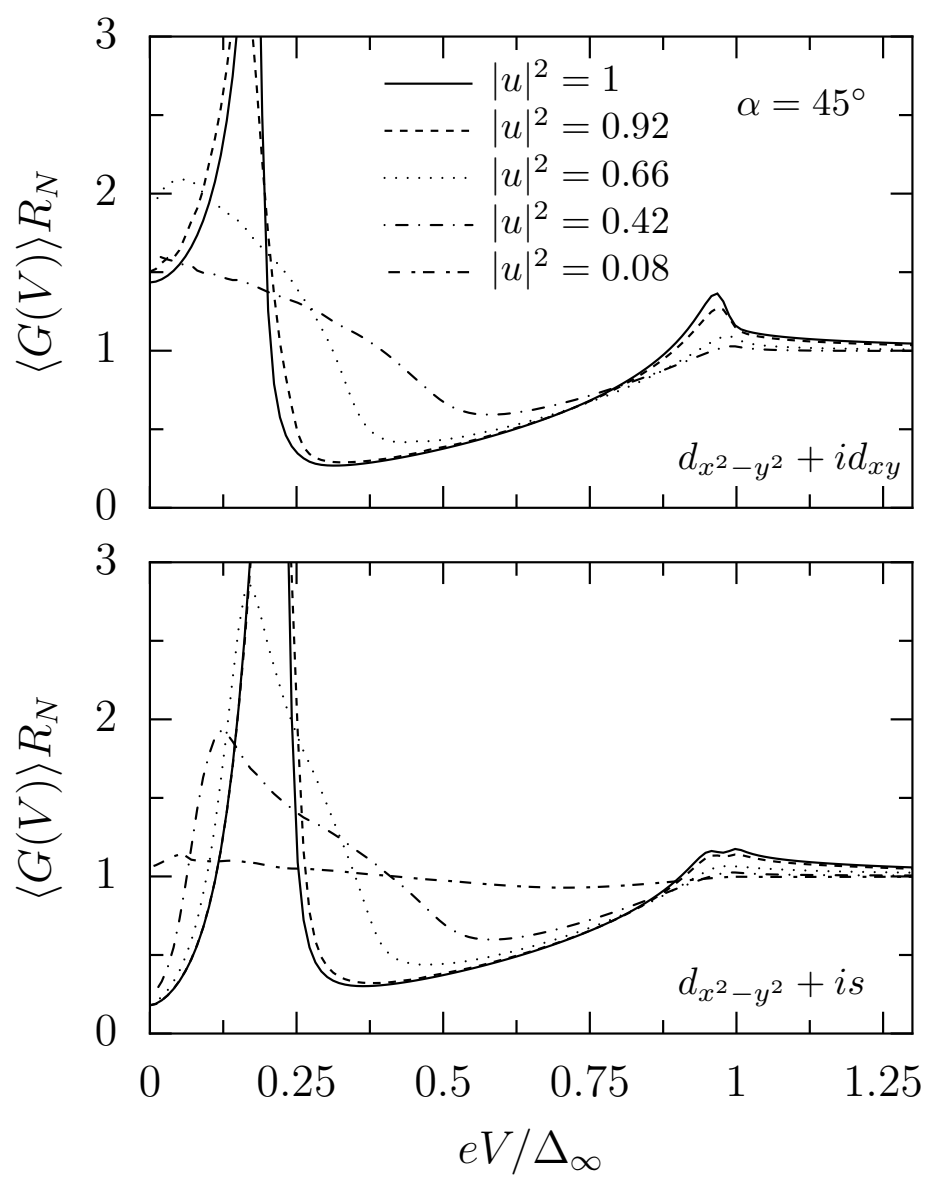

Fig. 4 Differential conductance versus voltage for $\alpha=45^{\circ}$, for subdominant pairing in the $s$-wave (lower panel) and $d_{x y}$-wave channel (upper panel).

Here the current behaves qualitatively different (Fig. 2) to what we found for subdominant $s$-wave pairing (Fig. 3); in particular here the current changes its sign at some distance from the surface. This is related to the fact that a mixed $d_{x^{2}-y^{2}}+i d_{x y}$ order parameter has a finite magnetic moment, which is not the case for a $d_{x^{2}-y^{2}}+i s$ order parameter.

A crucial difference occurs for rough surfaces: The $d_{x y}$-wave component is not only suppressed by the dominant order parameter but, as it is anisotropic, also by the surface roughness. Therefore the subdominant admixture decreases rapidly with increasing surface roughness; we find a full suppression already for $|u|^{2} \lesssim 0.42$ (Fig. 3).

We now study the situation when the order parameter is rotated by only $24^{\circ}$ with respect to the surface normal, and a subdominant $d_{x y}$-wave pairing is present. The crucial difference to the previous cases is that the dominant order parameter is not completely suppressed by the specular surface. We also find a finite value of the subdominant order parameter but with a relative phase $\varphi \approx 0$; i.e. the admixture is mainly real-valued, and the time-reversal symmetry-breaking imaginary part is negligible (Fig. 5). Therefore no splitting of the zero-bias conductance peak can be observed (Fig. 6). The real-valued subdominant component leads to an effective rotation of the dominant order parameter such that the tilting angle is diminished. As a result the suppression of the order parameter at the surface is reduced; the differential conductance shows that spectral weight is shifted from lower to higher energies compared to the case 


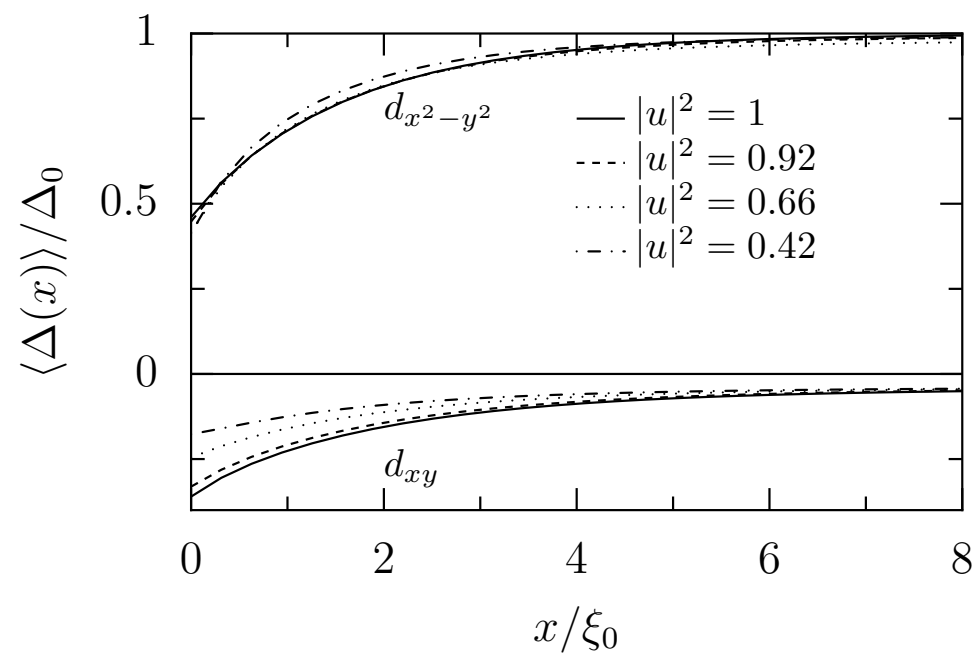

Fig. 5 Dominant $d_{x^{2}-y^{2}}$-wave order parameter with (real) subdominant $d_{x y}$-wave component, for $\alpha=$ $24^{\circ}$ at $T=0.1 T_{c} ; \Delta_{0}=2.14 T_{c}$, and $\xi_{0}=v_{F} / \pi \Delta_{0}$.

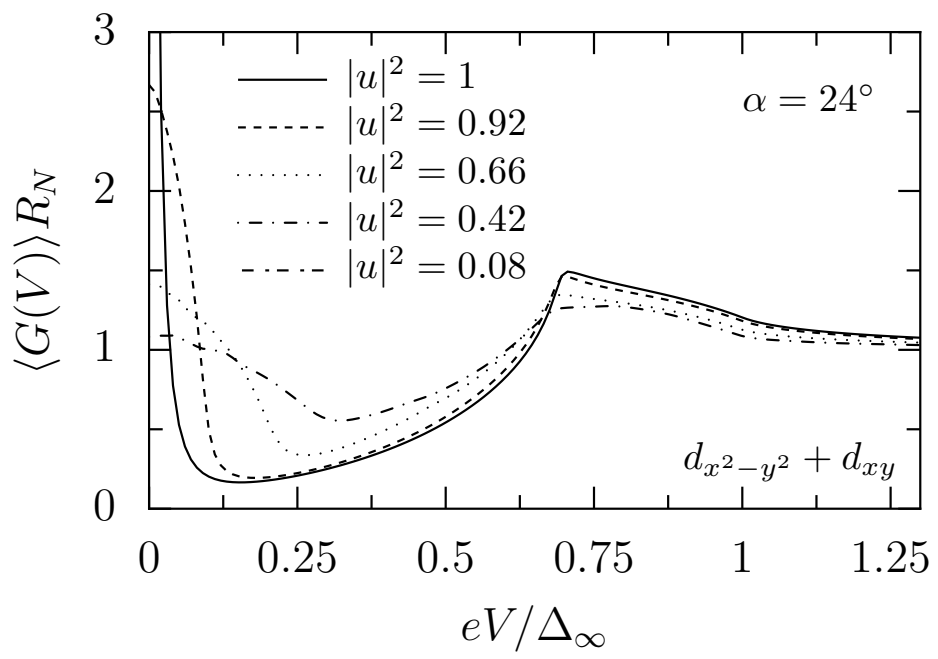

Fig. 6 Differential conductance versus voltage for $\alpha=24^{\circ}$, for subdominant pairing in the $d_{x y}$-wave channel. Note, that for the clean case $\left(|u|^{2}=1\right)$ the zero-bias conductance peak has been broadend by adding a finite imaginary part to the energy.

without subdominant admixture. For subdominant $s$-wave pairing a similar effect occurs. For a $45^{\circ}$-tilting of the order parameter no real-valued admixture develops due to the symmetry of the system.

In conclusion we have shown that, for a $45^{\circ}$-rotated order parameter, surface roughness suppresses the subdominant admixture in the $d_{x y}$-wave as well as in the $s$-wave case. We have also found that the subdominant component may vanish completely for very strong roughness. As expected the $d_{x y}$-wave component is more strongly affected by roughness than an $s$-wave component. For a tilting angle $\alpha<$ $45^{\circ}$, a real-valued admixture occurs as well; the order parameter is modified so that its suppression is weakened. Our results imply that surface roughness plays a crucial role for the experimental observation 
of subdominant pairing. In particular the roughness must be small enough to detect the splitting of the zero-bias conductance peak.

Acknowledgements Helpful discussions with U. Eckern and P. Schwab are gratefully acknowledged. This work was supported by the Deutsche Forschungsgemeinschaft (project: LU 863/1-1).

\section{References}

[1] M. Matsumoto and H. Shiba, J. Phys. Soc. Jpn. 64, 3384 (1995).

[2] M. Matsumoto and H. Shiba, J. Phys. Soc. Jpn. 64, 4867 (1995).

[3] M. Fogelström, D. Rainer, and J. A. Sauls, Phys. Rev. Lett. 79, 281 (1997).

[4] M. Fogelström, D. Rainer, and J. A. Sauls, cond-mat/0302197

[5] M. Covington et al., Phys. Rev. Lett. 79, 277 (1997).

[6] Y. Dagan and G. Deutscher, Phys. Rev. Lett. 87, 177004 (2001).

[7] L. Alff et al., Phys. Rev. B 55, 14757 (1997).

[8] I. Iguchi, W. Wang, M. Yamazaki, Y. Tanaka, and S. Kashiwaya, Phys. Rev. B 62, R6131 (2000).

[9] N.-C. Yeh et al., Physica C 364-365, 450 (2001).

[10] J. J. Betouras and R. Joynt, Phys. Rev. B 57, 11752 (1998).

[11] D. B. Bailey, M. Sigrist, and R. B. Laughlin, Phys. Rev. B 55, 15239 (1997).

[12] G. Eilenberger, Z. Phys. 214, 195 (1968).

[13] J. Rammer and H. Smith, Rev. Mod. Phys. 58, 323 (1986).

[14] T. Lück, U. Eckern, and A. Shelankov, Phys. Rev. B 63, 64510 (2001).

[15] A. Zaitsev, Sov. Phys. JETP 59, 1015 (1984).

[16] K. Yamada, Y. Nagato, S. Higashitani, and K. Nagai, J. Phys. Soc. Jpn. 65, 1540 (1996).

[17] Y. S. Barash, A. A. Svidzinski, and H. Burkhardt, Phys. Rev. B 55, 15239 (1997).

[18] A. Poenicke, Y. S. Barash, C. Bruder, and V. Istyukov, Phys. Rev. B 59, 7102 (1999).

[19] A. Poenicke, M. Fogelström, and J. A. Sauls, Physica B 284-288, 589 (2000).

[20] A. Shelankov and M. Ozana, Phys. Rev. B 61, 7077 (2000).

[21] T. Lück, P. Schwab, U. Eckern, and A. Shelankov, cond-mat/0310250

[22] D. Rainer, H. Burkhardt, M. Fogelström, and J. A. Sauls, J. Phys. Chem. Solids 59, 2040 (1998). 